Portland State University

PDXScholar

\title{
Characterization of Retinol Stabilized in Phosphatidylcholine Vesicles With and Without Antioxidants
}

Yekaterina G. Chmykh

Portland State University

Jay Nadeau

Portland State University, nadeau@pdx.edu

Follow this and additional works at: https://pdxscholar.library.pdx.edu/phy_fac

Part of the Physics Commons

Let us know how access to this document benefits you.

\section{Citation Details}

Chmykh, Y. G., \& Nadeau, J. L. (2020). Characterization of Retinol Stabilized in Phosphatidylcholine Vesicles with and without Antioxidants. ACS omega, 5(29), 18367-18375.

This Article is brought to you for free and open access. It has been accepted for inclusion in Physics Faculty Publications and Presentations by an authorized administrator of PDXScholar. Please contact us if we can make this document more accessible: pdxscholar@pdx.edu. 


\title{
Characterization of Retinol Stabilized in Phosphatidylcholine Vesicles with and without Antioxidants
}

\author{
Yekaterina G. Chmykh and Jay L. Nadeau*
}

Cite This: ACS Omega 2020, 5, 18367-18375

Read Online

ABSTRACT: Retinol stability has been reported to be improved by encapsulation in liposomes, both with and without cholesterol. However, this improvement is limited because of lipid peroxidation. In this study, we compare the stability of retinol in phosphatidylcholine liposomes under ultraviolet (UV) light or standard room air, with and without the addition of antioxidants. Both butylated hydroxytoluene (BHT) and a proprietary mix (StoppOx) improved the shelf stability from $<10$ to over $30 \mathrm{~d}$. The addition of cholesterol had no effect. Fluorescence imaging showed a heterogeneous distribution of retinol within the vesicles, including within the aqueous layer. Fluorescence lifetimes were equally heterogeneous. Under UV irradiation, StoppOx protected

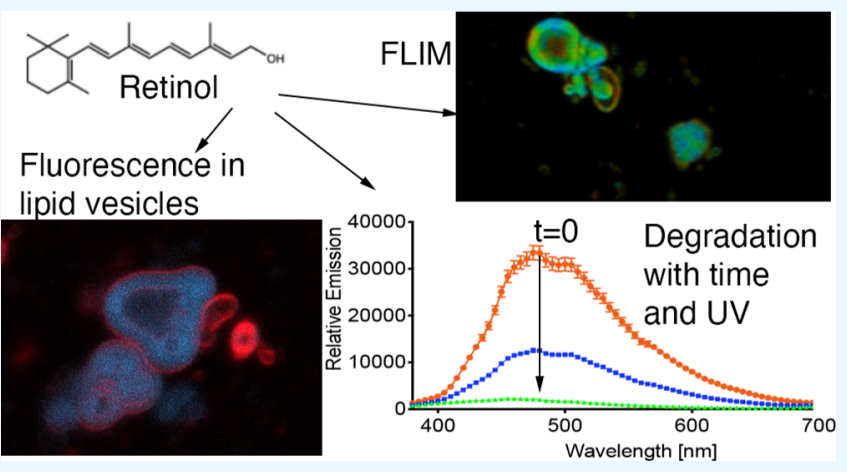
retinol for significantly longer than $\mathrm{BHT}$ and via different mechanisms. This suggests that natural antioxidants work well to improve the retinol stability, but that further work to determine the optimal vesicle structure remains to be performed.

\section{INTRODUCTION}

Retinol (vitamin A) is used in pharmaceuticals and cosmetics for dermal applications to correct hyperpigmentation, reduce the appearance of fine lines, and treat acne and atopic dermatitis. Both prescription and nonprescription topical creams have shown to result in clinically significant improvement of the appearance of aged and photodamaged skin. ${ }^{1-5}$ Creating stable formulas for consumer use, however, remains a barrier as retinol is highly sensitive to light and oxygen. UV light causes degradation into a variety of potentially harmful products. Photoirradiation of retinol by UV light $(254 \mathrm{~nm})$ in an oxygenated ethanolic solution has been shown to produce retinal, $\mathrm{ROH}$ 5,6-epoxide, 5,8-epoxyretinol, and 13,14-epoxyretinol. Irradiated retinol can also activate photosensitizers, such as chlorophyll, rose bengal, or riboflavin, with drastic alteration of the resulting photoproducts. ${ }^{6}$ Depending upon the conditions, reactive oxygen species (ROS), including singlet oxygen, may be produced. ${ }^{7-9}$ Endogenous photosensitizers in cells result in the production of a large number of photoproducts not predicted from simple solutions; these products may damage DNA and cell membranes directly or indirectly (Scheme 1). Because these effects are well known, most retinoid preparations are intended as "night creams" with use during the day not recommended. Preparations must be protected from light, refrigerated, and protected with an antioxidant preservative, usually butylated hydroxytoluene (BHT). Most retinol preparations sold to cosmetics manufacturers are preserved with BHT.
Recent concern over possible adverse health effects of BHT has led to its removal from food products by major manufacturers. General Mills removed BHT from its packaged cereals in 2015, and Kellogg's has offered a variety of BHT-free products, although they have planned to continue using BHT in their cereals. BHT and butylated hydroxyanisole are widely used antioxidant food additives that have been extensively studied for their safety; although they have not been associated with cancer, ${ }^{10}$ consumer concerns persist. Several studies have reported pulmonary toxicity in mice resulting from oral or topical exposure to BHT. ${ }^{11,12}$ In 2007, a study suggested that BHT may aggravate allergic responses. ${ }^{12}$

Although concentrations found in cosmetics are reported to be safe for topical use, ${ }^{13}$ there is increasing interest in BHTfree topical retinol products. Methods of stabilization include natural antioxidants, encapsulation, or some combination of both. Encapsulation materials include different types of lipids, chitosan, pectin, and zein particles. ${ }^{14-20}$ Lipid particles have been shown to improve the skin uptake of retinol compared to control solutions, ${ }^{21,22}$ but simple lipid encapsulation can increase the shelf life only to a limited degree because the

Received: May 6, 2020

Accepted: June 26, 2020

Published: July 10, 2020 
Scheme 1. Reaction Pathways of Liposome-Encapsulated Retinol $^{a}$

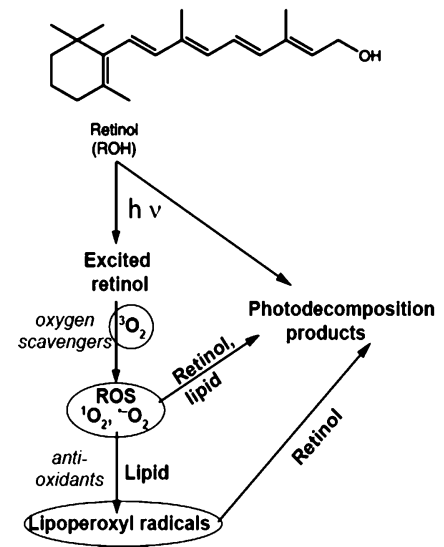

${ }^{a}$ Photoexcitation may lead to direct breakdown into retinal, epoxides, or other products, or may lead to excited-state retinol which interacts with oxygen to produce ROS, including singlet oxygen and superoxide; these ROS may break down retinol and lipids; lipoperoxyl radicals further degrade retinol. The ellipses and italicized labels indicate where the degradation reactions may be inhibited: oxygen scavengers interact directly with molecular oxygen and prevent the formation of ROS, while antioxidants react with ROS and other radicals to compete with their reaction with retinol.

lipids themselves are vulnerable to oxidation. Lipid microcapsules oxidize more rapidly than bulk lipids because of the increased surface area and preferential localization of lipid hydroperoxides at the oil-water interface; lipid oxidation may cause increased degradation of encapsulated products. ${ }^{23,24}$ Retinoids can photosensitize the formation of lipid hydroperoxides in a UV dose-dependent fashion. ${ }^{25}$

A number of studies use antioxidants, including BHT and metal chelates, to improve the stability of lipid-encapsulated pharmaceuticals. $^{26-28}$ Other studies have looked at oxygen scavengers, which interact directly with oxygen rather than with secondary products of oxidation reactions. Oxygen scavengers include sodium sulfite $\left(\mathrm{Na}_{2} \mathrm{SO}_{3}\right)$, a combination of glucose oxidase and catalase ( $\mathrm{GOx}+$ catalase) and ascorbic acid (AA). ${ }^{23}$

The purpose of this study was to characterize retinol encapsulated in phosphatidylcholine (PC) multilamellar vesicles. We characterized the liposomes using confocal microscopy and fluorescence lifetime imaging (FLIM). Imaging demonstrated an unusual pattern of retinol distribution throughout the liposomes, including within the aqueous phase. The distribution of retinol fluorescence was found to be diffuse and dispersed, not overlapping the staining seen using membrane-targeting dyes such as FM 1-43. Retinol fluorescence lifetimes varied from liposome to liposome and among domains in individual liposomes, reflecting the environmental effects of liposome size and lamellarity on fluorescence lifetimes.

Retinol was encapsulated in PC with and without preservatives (BHT or a "natural" lipophilic preparation $[$ StoppOx] $)$. Using the native fluorescence of retinol, the

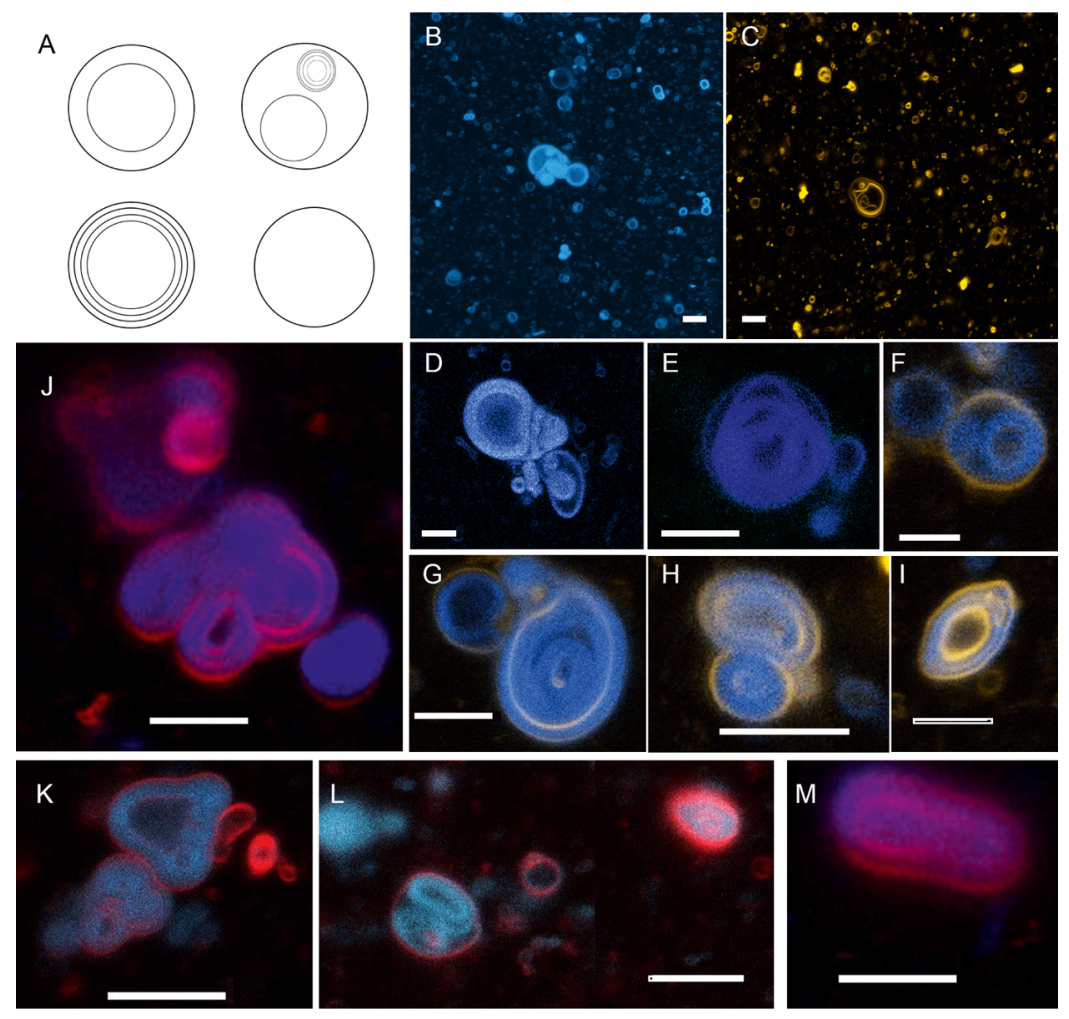

Figure 1. Confocal images of PC-retinol liposomes. Scale bars $=5 \mu \mathrm{m}$. The colors represent a pseudocolor code as follows: blue, excitation $405 \mathrm{~nm}$, emission 420-480 nm; yellow, excitation $488 \mathrm{~nm}$, emission 495-550 nm; and red, excitation $543 \mathrm{~nm}$ and emission $680 \mathrm{~nm}$ longpass. (A) Schematic of some possible configurations of multilamellar liposomes. (B) Image of retinol-only liposomes. (C) Image of PC liposomes containing retinol and labeled with FM 1-43, showing the dye-only and lack of bleed-through in the yellow pseudo-colored channel. (D,E) Zoom of single liposomes with retinol only. (F-I) Zoom of single liposomes with retinol and FM 1-43. (J-L) Zoom of single liposomes with retinol and FM 4-64. (M) Single liposome containing 10\% cholesterol, with retinol and FM 4-64. 

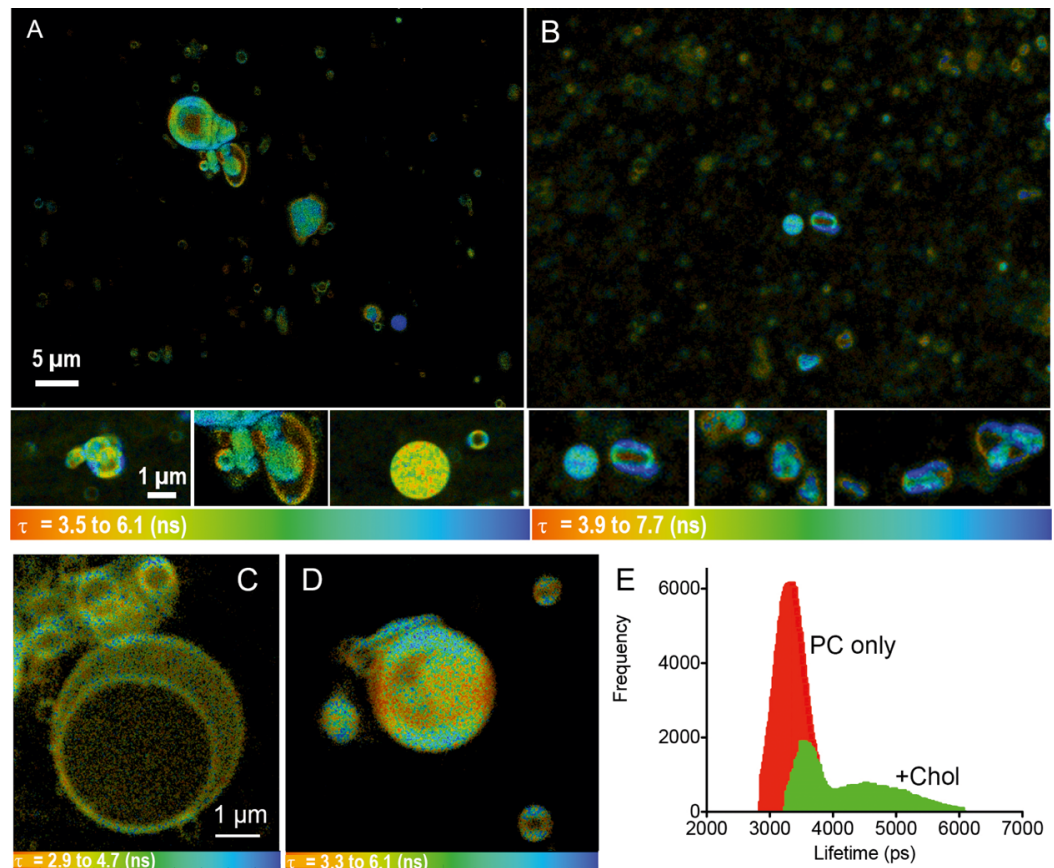

Figure 2. Fluorescence lifetime images of retinol in vesicles. Excitation was $730 \mathrm{~nm}$ and emission was $500-550 \mathrm{~nm}$. Images are color-coded according to the lifetime, as shown in the color bar. (A) PC-retinol. (B) PC-retinol-cholesterol. (C) Zoom in of a single PC-retinol vesicle. (D) Zoom in of a single PC-retinol-cholesterol vesicle, showing areas suggesting domains. (E) Histogram of lifetimes of all vesicles in panels A (PC only) and B (+Chol), showing a single discrete peak in the PC-only liposomes and two peaks in the liposomes containing cholesterol.

stability was monitored for several weeks under ambient conditions, or for $20 \mathrm{~min}$ under UV irradiation. Liposomes with and without $10 \%$ cholesterol were also tested under these conditions because addition of cholesterol has been reported to improve the stability of liposome-encapsulated retinol. ${ }^{29}$

Retinol photoluminescence has been well studied and is an established method of determining retinol concentration. ${ }^{30,31}$ The absorption band at $325 \mathrm{~nm}$ with an emission at $\sim 490 \mathrm{~nm}$ is due to a $\pi-\pi$ transition of the retinoid's polyene chain. As the retinol degrades, this fluorescence disappears. Multilamellar vesicles were more effective than unilamellar vesicles in protecting retinol, so raw vesicles without extrusion or freeze-thaw, which makes vesicles uniform and unilamellar, ${ }^{32}$ were selected for a long-term study. We found that StoppOx was as effective as BHT in preserving retinol under both ambient conditions and UV light. Liposome preparations containing StoppOx were stable for at least 1 month under ambient light and temperature. A biphasic-modified Gompertz growth model was used to fit the decay. When exposed to UV light, retinol fluorescence degraded over a time course of 20 min, with liposomes containing StoppOx being more stable than liposomes with BHT. The photodegradation curves could be fit to a mix of first- and second-order processes, which indicated that StoppOx protected the liposomes from firstorder photobleaching, while BHT did not.

\section{RESULTS AND DISCUSSION}

Encapsulation Efficiency. High-performance liquid chromatography (HPLC) was used only to estimate the encapsulation efficiency. The supernatant from retinolcontaining liposomes had retinol concentrations below the limits of detection $(<5 \mathrm{IU} / \mathrm{mL}$ retinol), indicating an encapsulation efficiency of $\geq 98.9 \%$. Previous studies have seen only small changes in efficiency $(<1 \%)$ due to $\mathrm{pH}$ changes (between 3 and 11) and increased mass loading, ${ }^{14,18}$ so we did not vary these parameters for our studies.

Vesicle Characterization. The vesicle shape, size, and lamellarity were investigated by confocal microscopy, with and without an additional membrane dye ( $\mathrm{N}$-(3-triethylammoniumpropyl)-4-(4-(dibutylamino)styryl)pyridinium dibromide [FM 1-43] or N-(3-triethylammoniumpropyl)-4-(6-(4(diethylamino)phenyl)hexatrienyl)pyridinium dibromide [FM 4-64]) (Figure 1). PC liposomes containing retinol had a mean ( \pm standard deviation) diameter of $4 \pm 3 \mu \mathrm{m}$ (range: $1.2-56$ $\mu \mathrm{m}, N=909$ ), as measured from the microscopic images. With $10 \%$ cholesterol, the mean size was reduced slightly, with a marked reduction in the largest vesicles (mean diameter: $3 \pm 3$ $\mu \mathrm{m}$, range: $1.2-24 \mu \mathrm{m}, N=553$ ). FM 4-64 emits in the far-red and was compared with the greener FM 1-43 to ensure that there was no channel bleed-through from retinol emission (see Figure S1 for spectra). Retinol emission appeared diffuse, mostly near the edges of the liposome but between layers as well, in contrast to the sharp membrane labeling seen with the FM dyes (Figure $1 \mathrm{~A}-\mathrm{C}$ ). Zoomed images of individual vesicles showed some retinol fluorescence limited to lipid bilayers, but a substantial amount occurring throughout subcompartments of the liposome or even the entire liposome (Figure 1D,E). Colabeling with FM dyes illustrated the thickness of the bilayer and emphasized the presence of the retinol between layers (Figure $1 \mathrm{~F}-\mathrm{L}$ ). The addition of cholesterol led to slightly smaller vesicles but no qualitative change in the fluorescence distribution (Figure 1M). This appearance is consistent with the ability of retinol to rapidly transfer between and among lipid bilayers, as has been observed by bulk spectroscopy and radiolabeling. A time constant of $<30 \mathrm{~s}$ has been reported for transfer between bilayers, indicating that despite its hydrophobic nature, retinol is able to dissociate from lipids and travel through the aqueous phase without the assistance of shuttle proteins. ${ }^{33-35}$ 

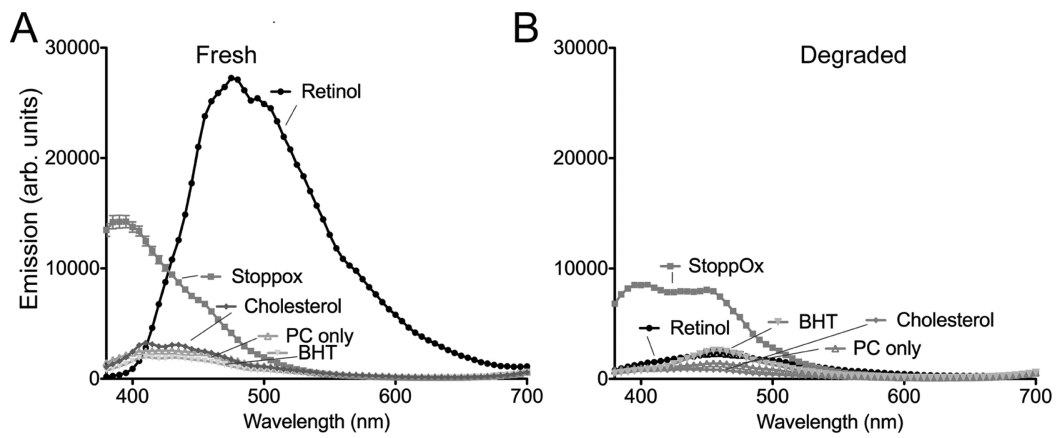

Figure 3. Fluorescence emission spectra of retinol, antioxidants, and lipids with excitation at $348 \mathrm{~nm}$. (A) Freshly prepared solutions. Retinol dominates the emission; to avoid the StoppOx signal, peaks were measured at $505 \mathrm{~nm}$ for decay curves. (B) In degraded solutions, the emission of StoppOx dominates, although it is less than that seen in fresh solutions.
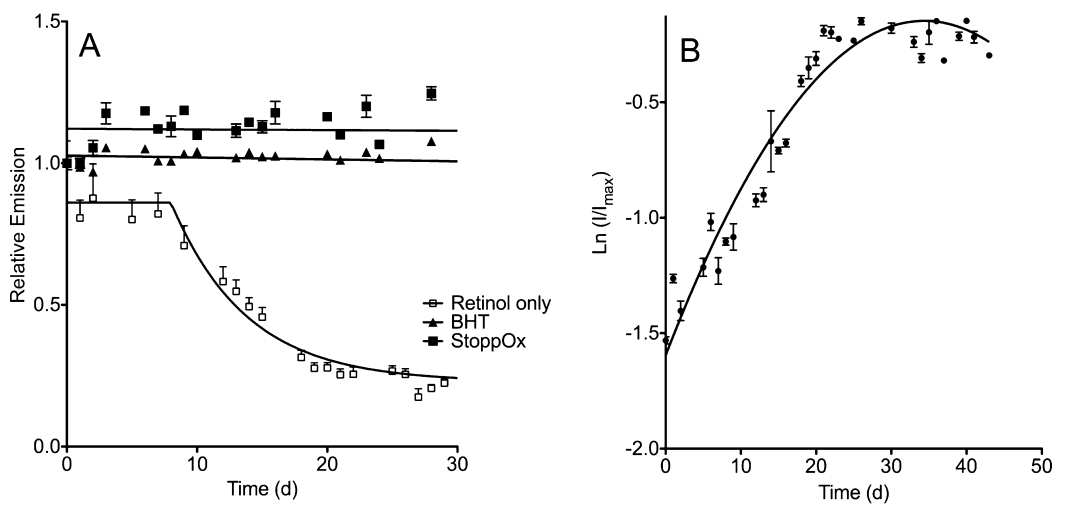

Figure 4. Retinol stability in liposomes with or without BHT or StoppOx. (A) Relative emission. The data points show fluorescence emission at $505 \mathrm{~nm}$ normalized to time $t=0$. For the first $30 \mathrm{~d}$, no significant decay of retinol in BHT- or StoppOx-containing liposomes was seen. The points show relative emission \pm SEM for two independent studies, each run in triplicate; the lines are linear fits with the slope insignificantly different from 0 . Retinol-only showed a plateau for $8.0 \pm 0.6 \mathrm{~d}$, followed by an exponential decay with $k=0.17 \pm 0.03$. (B) Natural log of emission normalized to its maximum value. The emission of antioxidant-protected liposomes relative to retinol-only liposomes showed stability for $30 \mathrm{~d}$ followed by slow decay, which could be fit to the biphasic model discussed in the text.

FLIM images also showed significant heterogeneity in the liposome size, lamellarity, and retinol content. The fluorescence lifetime of retinol alone excited at $730 \mathrm{~nm}$ was $3.4 \pm 0.3$ ns in our experiments, with significant variation among vesicles and different layers of the same vesicle (Figure 2A). In the presence of $10 \%$ cholesterol, areas of longer lifetime were apparent in the images; the lifetime showed two distinct distributions and could not be fit to a single decay. One component was equivalent to that of the PC-only vesicles ( 3.5 $\pm 0.4 \mathrm{~ns})$, while the other was longer $(4.5 \pm 0.3 \mathrm{~ns})$. Many vesicles appeared similar to the PC-only vesicles, where a few were clearly dominated by the longer lifetime, suggesting a heterogeneous distribution of the cholesterol (Figure 2B). Closeups of single vesicles showed homogeneous lifetimes within and between bilayers of PC-only vesicles (Figure 2C), while longer lifetime domains could be seen in cholesterolcontaining vesicles (Figure 2D). Lifetime histograms showed a single distinct lifetime for PC-only vesicles, but two distinct components for cholesterol-containing vesicles; the strength of this second component varied from sample to sample, with the data shown representing one chosen field of view (Figure $2 \mathrm{E}$ ). These findings are consistent with previous results, which have shown that retinol photophysical properties are sensitive to the molecular conformation and environment, ${ }^{36}$ with reported lifetimes of $2.3 \mathrm{~ns}$ in glycerol, $4.2 \mathrm{~ns}$ in hexane, and 6-8 ns when bound to proteins such as albumin or retinol-binding protein. $^{37}$
All imaging experiments were performed on fresh samples. As retinol degraded, it lost its fluorescence. No emission was observed from vesicles stored for $30 \mathrm{~d}$ or exposed to UV light for $20 \mathrm{~min}$ (not shown). The addition of KI did not quench the vesicle fluorescence (Figure S2).

Because of the high degree of heterogeneity, we also prepared batches of unilamellar vesicles by freeze-thaw. These vesicles were uniform in size and lamellarity (data not shown). However, retinol in these vesicles was less stable than that in the multilamellar vesicles, so their use was abandoned. Their fluorescence was also not substantially affected by KI (Figure S3).

Spectroscopy and Stability Analysis. Retinol in PC vesicles showed a broad fluorescence emission peak at $490 \mathrm{~nm}$ when excited at $348 \mathrm{~nm}$ (Figure 3A). Both BHT and StoppOx showed some degree of fluorescence at this excitation wavelength, but it was significantly blue-shifted relative to that of retinol, so that a wavelength could be chosen that would indicate retinol only in both fresh and degraded solutions $(505 \mathrm{~nm})$. It was important to monitor the fluorescence changes of the antioxidant-only controls as solutions degraded because the antioxidant compounds showed shifts in emission with time and light exposure (Figure 3B).

Long-term stability analyses showed that both BHT and StoppOx prevented retinol degradation in liposomes for at least 30 days before the loss of emission began. There was no 

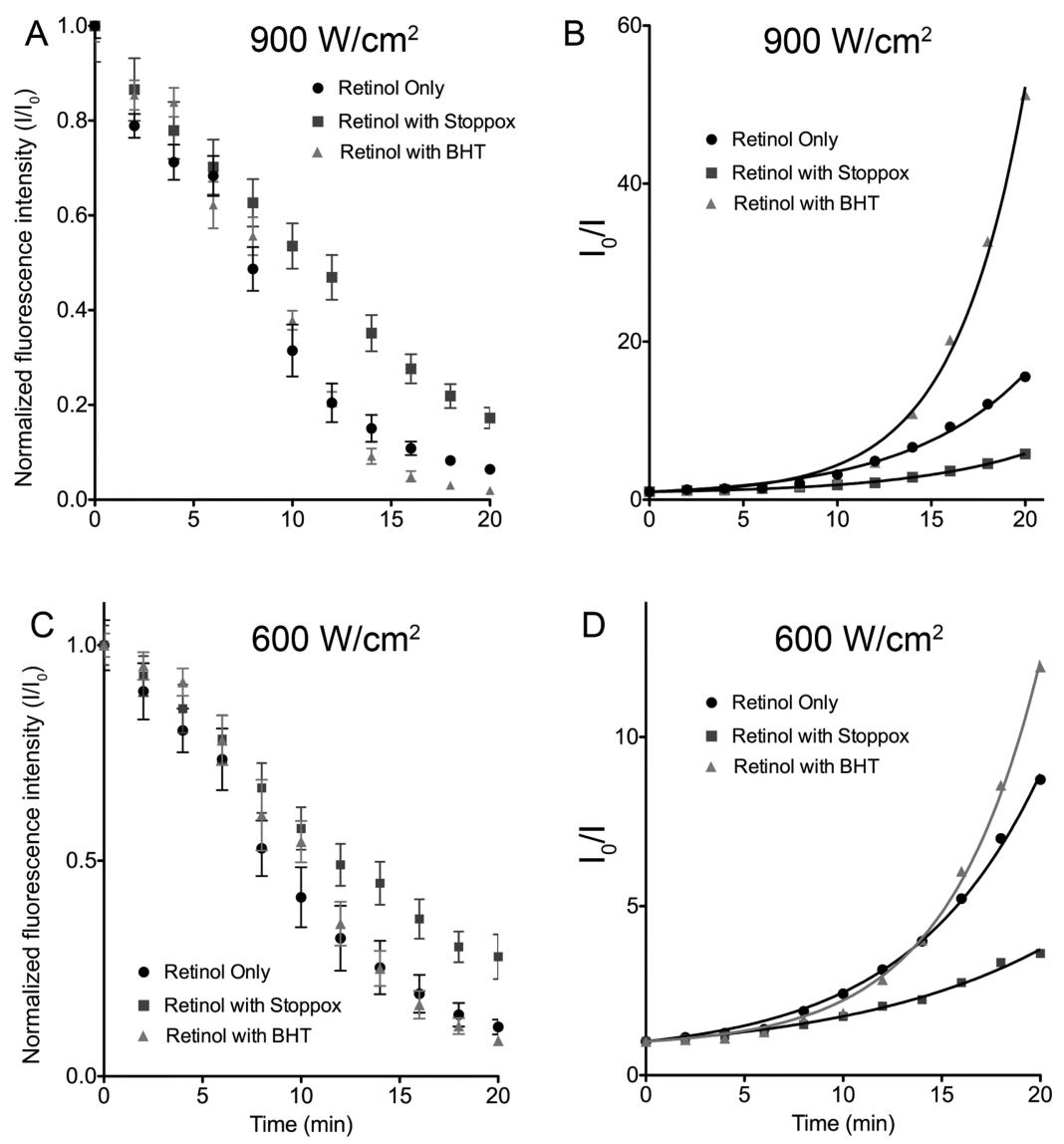

Figure 5. Fluorescence decay as a function of time under UV irradiation. (A) High power irradiation, fluorescence intensity normalized to time 0 for retinol only, retinol + BHT, and retinol + StoppOx. (B) Reciprocal of normalized intensity for high power irradiation with fit to eq 4a (fit parameters in Table 1). (C) Low power irradiation, fluorescence intensity normalized to time 0 for retinol only, retinol $+\mathrm{BHT}$, and retinol + StoppOx. (D) Reciprocal of normalized intensity for low power irradiation with fit to eq 4a (fit parameters in Table 1).

significant difference between BHT and StoppOx (Figure 4A). Liposomes containing retinol only, without antioxidants, were stable for $\sim 8 \mathrm{~d}$ before the signal began decaying exponentially (Figure 4A).

After $30 \mathrm{~d}$, the antioxidant-containing liposomes began to show a loss of signal in a nonexponential fashion (Figure 4B). The fluorescence of StoppOx-containing liposomes normalized to that of the controls could be fit to a modified Gompertz equation that identifies a turning point $t_{\max }$ where the sigmoidal growth decays. ${ }^{38}$ The full equation for the model may be written as

$$
\ln \left(\frac{I}{I_{\max }}\right)=\mu_{\min }\left(t-t_{0}\right)-\frac{\mu_{\min }}{k}\left(1-\mathrm{e}^{-k\left(t-t_{\max }\right)}\right)
$$

where $I / I_{\max }$ is the fluorescence intensity normalized to its maximum value; $t$ is the time; $t_{\max }$ is the time at which the maximum signal occurs; $k$ is a rate constant that determines the rate at which the growth rate approaches the final net growth rate, $\mu_{\min }$ at long times. The advantage of this model is that it is an equation with a single nonlinear parameter, $k$. This fit then yields a meaningful interpretation of the curve. It may also be seen that at values of $\mathrm{t}$ close to $t_{\max }$, the curve becomes a parabola

$$
\ln \left(\frac{I}{I_{\max }}\right) \approx \frac{k \mu_{\min }}{2}\left(t-t_{\max }\right)^{2}
$$

allowing for the choice of initial fit values of the parameters or for an approximate fit of $I$ to a Gaussian function. Approximation of the function as a Gaussian did not change the quality of the fit for our data, making this a very simple model. The fit values for two independent 42 -day studies were consistent, and are plotted together in Figure 4B. Fit values were $t_{\max }=31.5 \pm 0.5$ days and $k \mu_{\min }=0.0033 \pm 0.0002$ days $^{-2}$.

Photolysis. Liposomes with retinol only and retinol plus BHT or StoppOx were exposed to UV irradiation at 900 and $600 \mu \mathrm{W} / \mathrm{cm}^{2}$. Two independent preparations of vesicles were prepared in 6-fold replicates each, and fluorescence emission was measured every $2 \mathrm{~min}$ for $20 \mathrm{~min}$. The results of the two independent trials were consistent; one is shown in Figure 5A. It can be seen that while StoppOx protected retinol from photolysis under these conditions, the presence of BHT led to increased degradation at long times $\left(>10 \mathrm{~min}\right.$ for $900 \mathrm{~W} / \mathrm{cm}^{2}$ and $>15 \mathrm{~min}$ for $600 \mathrm{~W} / \mathrm{cm}^{2}$ ).

These data could be fit to a model of photobleaching, as described previously for photosensitizers and other fluorophores. ${ }^{39-41}$ The photobleaching reactions are expressed as a composite of reactions with a first-order term and a secondorder term

$$
\frac{\mathrm{d}[\mathrm{R}]}{\mathrm{d} t}=-C_{1}[\mathrm{R}]-C_{2}[\mathrm{R}]^{2}
$$

This may be rewritten as 
Table 1. Fit Parameters for Photolysis to eq $4 a^{a}$

$\begin{array}{lcccc} & C_{1} & C_{2} R_{0} & R_{2} & \text { change in } C_{2} \\ \text { retinol only: high } & 0.156 \pm 0.009 & -0.32 \pm 0.01 & 0.9936 & \mathrm{~N} / \mathrm{A} \\ \text { retinol only: low } & 0.149 \pm 0.005 & -0.58 \pm 0.04 & 0.9975 & \mathrm{~N} / \mathrm{A} \\ \text { retinol + StoppOx: high } & 0.149 \pm 0.004 & -0.74 \pm 0.02 & 0.9981 & \mathrm{~N} / \mathrm{A} \\ \text { retinol + StoppOx: low } & 0.098 \pm 0.008 & -0.56 \pm 0.08 & 0.9923 & \\ \text { retinol +BHT: high } & 0.26 \pm 0.01 & -0.74 \pm 0.06 & 0.9941 & \mathrm{~N}<0.0005 \\ \text { retinol +BHT: low } & 0.211 \pm 0.007 & -0.83 \pm 0.02 & 0.9971 & P<0.0005\end{array}$

${ }^{a_{T}}$ The errors indicate uncertainty in the fit parameters. The last two columns indicate the significance of the change in each parameter relative to retinol-only, as measured by the extra sum-of-squares $\mathrm{F}$ test. $\mathrm{N} / \mathrm{A}=$ not applicable; NS = not significant.

$\frac{\mathrm{d}\left[\frac{1}{\mathrm{R}}\right]}{\mathrm{d} t}=C_{1}\left[\frac{1}{\mathrm{R}}\right]+C_{1} C_{2}$

The integrated rate equation is

$$
\frac{1}{R}+C_{2}=\left(\frac{1}{R_{0}}+C_{2}\right) \mathrm{e}^{C_{1} t}
$$

which can be written as

$$
\frac{1}{R}=\left(\frac{1}{R_{0}}+C_{2}\right) \mathrm{e}^{C_{1} t}-C_{2}
$$

or

$$
\frac{R_{0}}{R}=\left(1+C_{2} R_{0}\right) \mathrm{e}^{C_{1} t}-C_{2} R_{0}
$$

which is a fit with only two parameters, $C_{1}$ and $C_{2} R_{0}$. The observed fluorescence intensity is proportional to the light flux $\Phi$ as well as to $[R]$ in accordance with the equation

\section{$I \propto \Phi Q \varepsilon b[\mathrm{R}]$}

where $Q$ is the quantum yield of the molecule, $\varepsilon$ is the molar absorptivity, and $b$ is the pathlength. Thus, at a given intensity of excitation, the observed signal can be considered to be proportional to $[\mathrm{R}]$.

The data were excellent fits to eq $4 \mathrm{a}$ (Figure 5A,B) with fit values given in Table 1 . Several observations may be made: one, $C_{1}$ appears to depend upon light fluence only when StoppOx is present. $C_{1}$ is overall reduced by StoppOx but is increased by BHT. Both BHT and StoppOx increase $C_{2}$, which is $<0$. This means that $C_{2}$ does not represent second-order bleaching but second-order reconstitution of the fluorophore.

Studies with varying quenchers and oxygen concentrations have determined that the second-order reactions are due to the interaction of the fluorophore with species produced by neighboring fluorophores and then diffusing to the site of action. These species are unlikely to be singlet oxygen, which has a very short lifetime and thus radius of diffusion, but represent other radicals. ${ }^{39}$ In this case, retinol may be regenerated by direct reduction or interaction with molecular oxygen. Ignoring triplet interactions, the possible reactions are

$$
\begin{aligned}
& \mathrm{R}+h \nu \rightarrow \mathrm{R}^{*} \\
& \mathrm{R}^{*} \stackrel{k_{1}}{\rightarrow} \mathrm{X} \\
& \mathrm{R}^{*} \stackrel{k_{2}}{\rightarrow} \mathrm{R} \\
& \mathrm{R}^{*}+{ }^{3} \mathrm{O}_{2} \stackrel{k_{3}}{\rightarrow}{ }^{1} \mathrm{O}_{2}+\mathrm{R} \\
& { }^{1} \mathrm{O}_{2}+\mathrm{R} \stackrel{k_{4}}{\rightarrow} \mathrm{X}
\end{aligned}
$$

where $\mathrm{X}$ are nonfluorescent products.

BHT appears to increase the regeneration of retinol by second-order, light fluence-dependent processes. However, it increases direct, first-order photobleaching, rendering the molecule less stable to light after a period of several minutes. StoppOx reduces first-order photobleaching and makes this process more directly responsive to light fluence.

\section{CONCLUSIONS}

Retinol native fluorescence emission may be used to quantify the stability and to visualize the encapsulation in vesicles, with or without other dyes. A commercial blend of antioxidants, StoppOx, works as well as BHT for stabilizing retinol in liposomes for 30 days under ambient conditions; it is superior to BHT under UV light. Retinol degrades over a period of 3040 days under ambient conditions and over a period of $20 \mathrm{~min}$ when exposed to $900 \mathrm{~W} / \mathrm{cm}^{2} 312 \mathrm{~nm}$ light, with a mixture of first- and second-order processes. Further experiments with time-resolved spectra and deoxygenated solutions will elucidate these mechanisms further.

Although a significant number of studies have been done on the stability of retinol, the use of its native fluorescence emission for imaging is rare. The images here show some remarkable features. Retinol is found throughout the vesicles, including in the aqueous phase. Our finding that unilamellar vesicles were less stable than multilamellar vesicles may be related to this finding; retinol in unilamellar vesicles may concentrate predominantly in the aqueous phase and degrade more rapidly. The addition of aqueous antioxidants, such as AA, may further increase the stability in both unilamellar and multilamellar preparations.

\section{EXPERIMENTAL PROCEDURES}

High-Performance Liquid Chromatography. Using the standard method for testing vitamin A, HPLC-ultraviolet (UV) was performed by Intertek (Champaign, IL) (details are proprietary). For tests of encapsulation efficiency, liposomes were sedimented and the unencapsulated retinol remaining in the supernatant was quantified after dilution with vegetable oil. Encapsulation efficiency was estimated as 


$$
\% \text { ee }=100-\left(\left(\frac{\left(\frac{\mathrm{IU}}{3.33 \times 10^{3} \mathrm{mg}}\right) \times 10}{1.3 \mathrm{mg}}\right) \times 100 \%\right)
$$

where IU is the international unit used to measure vitamin quantities, $3.33 \times 10^{3} \mathrm{mg}$ is the conversion unit from IU to $\mathrm{mg}$, $1.3 \mathrm{mg}$ is the total amount of retinol encapsulated in the lipid solution, and the numeral 10 refers to the $10 \mathrm{~mL}$ of supernatant released into oil. The results were presented as $\mathrm{IU} /$ serving where a serving is $1 \mathrm{~g} / \mathrm{mL}$. The instrument limit of detection was $5 \mathrm{IU} / \mathrm{mL}$.

Chemicals. StoppOx ("Mixture from Sunflower Oil cold pressed organic, Tocopherols, bavarian hop extract and Ethylferulate") was purchased from All Organic Treasures GmbH. Crystalline all trans retinol, PC (from egg yolk, $\geq 40 \%$ enzymatic), chloroform, methanol, sodium chloride, glycine, cholesterol, pentetic acid (DTPA), potassium iodide (KI), and BHT were all purchased from Sigma-Aldrich and used as delivered. Glass beads, $5 \mathrm{~mm}$, case no. S800242, were purchased from Fisher Scientific.

Liposome Preparation. The method used for preparing liposomes was adapted from the literature. ${ }^{29}$ For stability studies, a $13 \mathrm{~mL}$ solution of chloroform and methanol (2:1 v/ v) was prepared with $130 \mathrm{mg}$ of $\mathrm{PC}$ in a $25 \mathrm{~mL}$ round-bottom flask. For trials assessing antioxidant properties, $13 \mu \mathrm{L}$ of StoppOx or $1.3 \mathrm{mg}$ of BHT was added. If needed, $10 \%$ cholesterol was added to the solution during this step as well. The organic solvents were removed under vacuum while being heated at $30{ }^{\circ} \mathrm{C}$ until only a lipid film was left in the flask. A 13 $\mathrm{mL}$ aqueous buffer $(10 \mathrm{mM}$ glycine and $0.115 \mathrm{M} \mathrm{NaCl}$, adjusted to $\mathrm{pH} 9$ with $\mathrm{NaOH}$ ) was added to the round-bottom flask along with glass beads to help with homogeneity. The flask was then placed under nitrogen and $1.3 \mathrm{mg}$ of retinol was added. The flask was then removed from nitrogen and the solution was mixed until homogeneous. The solution was stored in a closed $15 \mathrm{~mL}$ falcon tube at room temperature for immediate use or at $4{ }^{\circ} \mathrm{C}$, wrapped in a foil, for long-term storage.

For microscopy analyses and ultraviolet irradiation studies, retinol was added to the chloroform/methanol solution instead of the aqueous solution.

Absorbance and Emission. Fluorescence spectra, with excitation at $350 \mathrm{~nm}$ and emission at 400-704 $\mathrm{nm}$, were collected on a CLARIOstar plate reader (BMG Labtech) using epi-illumination in a 96-well black plate (Greiner). For quenching studies, $\mathrm{KI}$ was added from a $3 \mathrm{M}$ stock solution to the desired concentration.

Long-Term Stability. Liposome solutions were prepared and left at room temperature under ambient fluorescent room light. The fluorescence spectra were obtained for all the solutions in triplicates every 1-3 days, where the fluorescence emission was normalized to retinol-only liposome solutions.

UV Irradiation. Six-fold replicate samples in a 96-well quartz plate were exposed to a $312 \mathrm{~nm}$ UV transilluminator (Fisher Scientific, Pittsburgh, PA). Power at the sample was measured at $312 \mathrm{~nm}$ using a Coherent FieldMaster power meter with an IM2 UV sensor and was $\sim 900 \mu \mathrm{W} / \mathrm{cm}^{2}$. The power was modulated by placing the sample on a $3 \mathrm{~mm}$ thick glass plate, which reduced the power to $\sim 600 \mu \mathrm{W} / \mathrm{cm}^{2}$. The fluorescence spectrum with excitation at $348 \mathrm{~nm}$ and emission at $380-740 \mathrm{~nm}$ was recorded after successive 2 min UV exposure times.
Confocal Microscopy and FLIM. Confocal and lifetime images were obtained on a ZEISS LSM 880 confocal system with an Airyscan detector. All images shown were obtained using a $63 \times$ oil immersion objective $(\mathrm{NA}=1.4)$. Airyscan achieves super-resolution images by capturing 32 images of a single field of view across a specialized detector. Each image contains positional information that enables the reconstruction of a single super-resolution image. ${ }^{28,42}$ Channels collected were pseudo-colored as follows: Blue, excitation $405 \mathrm{~nm}$ (diode laser), emission 420-480; Green, excitation $488 \mathrm{~nm}$ (Ar ion), emission 495-550 nm; and Red, excitation $543 \mathrm{~nm}(\mathrm{He}-\mathrm{Ne})$, emission $680 \mathrm{~nm}$ longpass. FLIM microscopy was performed on the same microscope using a Becker \& Hickl SPC-150 FLIM module with a BIG2 detector. The dichroic in the BIG2 detector collected emissions in two channels: Channel, $1500-$ $550 \mathrm{~nm}$ (green) and Channel 2, 575-610 (red). Excitation for FLIM was provided by a Chameleon Ultra Ti:Sapphire laser (Coherent) operating at a repetitive rate of $80 \mathrm{mHz}$. The twophoton wavelength used for excitation was $730 \mathrm{~nm}$ based upon the literature. ${ }^{43,44}$ FLIM data were analyzed using SPCImage (Becker \& Hickl). Size distributions were obtained from confocal images using Fiji ${ }^{45}$ "analyze particles."

Curve Fitting. Graphing, calculation of standard deviations and standard errors, and curve fitting were performed using GraphPad Prism 8. Statistical differences between parameters were measured using the extra sum-of-squares $\mathrm{F}$ test and the AIC model.

\section{ASSOCIATED CONTENT}

\section{Supporting Information}

The Supporting Information is available free of charge at https://pubs.acs.org/doi/10.1021/acsomega.0c02102.

Spectra of lipids with dyes and results of KI quenching studies (PDF)

\section{AUTHOR INFORMATION}

\section{Corresponding Author}

Jay L. Nadeau - Department of Physics, Portland State University, Portland, Oregon 97201, United States; ○ orcid.org/0000-0001-5258-0076; Email: nadeau@pdx.edu

\section{Author}

Yekaterina G. Chmykh - Department of Physics, Portland State University, Portland, Oregon 97201, United States

Complete contact information is available at:

https://pubs.acs.org/10.1021/acsomega.0c02102

\section{Author Contributions}

The manuscript was written through contributions of all authors. All authors have given approval to the final version of the manuscript.

\section{Funding}

This work was supported by a contract from Marie-Veronique Inc. to Portland State University.

\section{Notes}

The authors declare no competing financial interest.

\section{ACKNOWLEDGMENTS}

We acknowledge expert technical assistance by staff in the Advanced Light Microscopy Core in the Department of Neurology and Jungers Center at Oregon Health and Science University. 


\section{ABBREVIATIONS}

PC, phosphatidylcholine; FLIM, fluorescence lifetime imaging microscopy; BHT, butylated hydroxytoluene; SEM, standard error of the mean

\section{REFERENCES}

(1) Bruce, S.; Barkovic, S. Open-Label Study Evaluating the AntiAging Effects of a 3-Product, 2-Step Retinol-Rejuvenation System Following 3 Months of Treatment in Subjects With Photodamage. J. Drugs Dermatol. 2017, 16, 23-28.

(2) Kafi, R.; Kwak, H. S.; Schumacher, W. E.; Cho, S.; Hanft, V. N.; Hamilton, T. A.; King, A. L.; Neal, J. D.; Varani, J.; Fisher, G. J.; Voorhees, J. J.; Kang, S. Improvement of naturally aged skin with vitamin A (retinol). Arch. Dermatol. 2007, 143, 606-612.

(3) Ho, E. T.; Trookman, N. S.; Sperber, B. R.; Rizer, R. L.; Spindler, R.; Sonti, S.; Gotz, V.; Mehta, R. A randomized, double-blind, controlled comparative trial of the anti-aging properties of nonprescription tri-retinol $1.1 \%$ vs. prescription tretinoin $0.025 \%$. J. Drugs Dermatol. 2012, 11, 64-69.

(4) Farris, P.; Zeichner, J.; Berson, D. Efficacy and Tolerability of a Skin Brightening/Anti-Aging Cosmeceutical Containing Retinol 0.5\%, Niacinamide, Hexylresorcinol, and Resveratrol. J. Drugs Dermatol. 2016, 15, 863-868.

(5) Varani, J.; Warner, R. L.; Gharaee-Kermani, M.; Phan, S. H.; Kang, S.; Chung, J.; Wang, Z.; Datta, S. C.; Fisher, G. J.; Voorhees, J. $\mathrm{J}$. Vitamin A antagonizes decreased cell growth and elevated collagendegrading matrix metalloproteinases and stimulates collagen accumulation in naturally aged human skin. J. Invest. Dermatol. 2000, 114, 480-486.

(6) Crank, G.; Pardijanto, M. S. Photooxidations and Photosensitized Oxidations of Vitamin-a and Its Palmitate Ester. J. Photochem. Photobiol., A 1995, 85, 93-100.

(7) Tolleson, W.; Cherng, S.-H.; Xia, Q.; Boudreau, M.; Yin, J.; Wamer, W.; Howard, P.; Yu, H.; Fu, P. Photodecomposition and phototoxicity of natural retinoids. Int. J. Environ. Res. Public Health 2005, 2, 147-155.

(8) Fu, P. P.; Cheng, S.-H.; Coop, L.; Xia, Q.; Culp, S. J.; Tolleson, W. H.; Wamer, W. G.; Howard, P. C. Photoreaction, phototoxicity, and photocarcinogenicity of retinoids. J. Environ. Sci. Health, Part C: Environ. Carcinog. Ecotoxicol. Rev. 2003, 21, 165-197.

(9) Fu, P. P.; Xia, Q.; Yin, J. J.; Cherng, S.-H.; Yan, J.; Mei, N.; Chen, T.; Boudreau, M. D.; Howard, P. C.; Wamer, W. G. Photodecomposition of vitamin A and photobiological implications for the skin. Photochem. Photobiol. 2007, 83, 409-424.

(10) Williams, G. M.; Iatropoulos, M. J.; Whysner, J. Safety assessment of butylated hydroxyanisole and butylated hydroxytoluene as antioxidant food additives. Food Chem. Toxicol. 1999, 37, 10271038.

(11) Yamaki, K.; Taneda, S.; Yanagisawa, R.; Inoue, K.; Takano, H.; Yoshino, S. Enhancement of allergic responses in vivo and in vitro by butylated hydroxytoluene. Toxicol. Appl. Pharmacol. 2007, 223, 164172.

(12) Waseem, M.; Kaw, J. L. Pulmonary effects of butylated hydroxytoluene in mice. Food Addit. Contam. 1994, 11, 33-38.

(13) Lanigan, R. S.; Yamarik, T. A. Final report on the safety assessment of BHT(1). Int. J. Toxicol. 2002, 21, 19-94.

(14) Argimoon, M.; Romero, M.; Miranda, P.; Mombruu, A. W.; Miraballes, I.; Zimet, P.; Pardo, H. Development and Characterization of Vitamin A-Loaded Solid Lipid Nanoparticles for Topical Application. J. Braz. Chem. Soc. 2017, 28, 1177-1184.

(15) Ding, Y.; Pyo, S. M.; Muller, R. H. smartLipids (R) as third solid lipid nanoparticle generation - stabilization of retinol for dermal application. Pharmazie 2017, 72, 728-735.

(16) Eskandar, N. G.; Simovic, S.; Prestidge, C. A. Chemical stability and phase distribution of all-trans-retinol in nanoparticle-coated emulsions. Int. J. Pharm. 2009, 376, 186-194.

(17) Huang, S.-J.; Sun, S.-L.; Chiu, C.-C.; Wang, L.-F. Retinolencapsulated water-soluble succinated chitosan nanoparticles for antioxidant applications. J. Biomater. Sci., Polym. Ed. 2013, 24, 315329

(18) Lee, S.-C.; Yuk, H.-G.; Lee, D.-H.; Lee, K.-E.; Hwang, Y.-I.; Ludescher, R. D. Stabilization of retinol through incorporation into Liposomes. J. Biochem. Mol. Biol. 2002, 35, 358-363.

(19) Ro, J.; Kim, Y.; Kim, H.; Park, K.; Lee, K.-E.; Khadka, P.; Yun, G.; Park, J.; Chang, S. T.; Lee, J.; Jeong, J. H.; Lee, J. Pectin Microand Nano-capsules of Retinyl Palmitate as Cosmeceutical Carriers for Stabilized Skin Transport. Korean J. Physiol. Pharmacol. 2015, 19, 5964.

(20) Pan, Y.; Tikekar, R. V.; Wang, M. S.; Avena-Bustillos, R. J.; Nitin, N. Effect of barrier properties of zein colloidal particles and oilin-water emulsions on oxidative stability of encapsulated bioactive compounds. Food Hydrocolloids 2015, 43, 82-90.

(21) Schafer-Korting, M.; Mehnert, W.; Korting, H. Lipid nanoparticles for improved topical application of drugs for skin diseases. Adv. Drug Delivery Rev. 2007, 59, 427-443.

(22) Trapasso, E.; Cosco, D.; Celia, C.; Fresta, M.; Paolino, D. Retinoids: new use by innovative drug-delivery systems. Expert Opin. Drug Delivery 2009, 6, 465-483.

(23) Chaudhari, A.; Nitin, N. Role of oxygen scavengers in limiting oxygen permeation into emulsions and improving stability of encapsulated retinol. J. Food Eng. 2015, 157, 7-13.

(24) Khanum, R.; Thevanayagam, H. Lipid peroxidation: Its effects on the formulation and use of pharmaceutical emulsions. Asian J. Pharm. Sci. 2017, 12, 401-411.

(25) Cherng, S.-H.; Xia, Q.; Blankenship, L. R.; Freeman, J. P.; Wamer, W. G.; Howard, P. C.; Fu, P. P. Photodecomposition of retinyl palmitate in ethanol by UVA light-formation of photodecomposition products, reactive oxygen species, and lipid peroxides. Chem. Res. Toxicol. 2005, 18, 129-138.

(26) Lin, C.-C.; Fujimoto, K.; Hwang, L. S. The Antioxidative Effect of Protein on the Hemoglobin Catalyzed Oxidation of Sardine Oil in an Emulsion System. J. Jpn. Soc. Food Sci. 1993, 40, 602-608.

(27) Ruben, C.; Larsson, K. Relations between Antioxidant Effect of Alpha-Tocopherol and Emulsion Structure. J. Dispersion Sci. Technol. 1985, 6, 213-221.

(28) Sims, R. J.; Fioriti, J. A.; Trumbetas, J. Effect of Sugars and Sugar Alcohols on Autoxidation of Safflower Oil in Emulsions. J. Am. Oil Chem. Soc. 1979, 56, 742-745.

(29) Lee, S.-C.; Lee, K.-E.; Kim, J.-J.; Lim, S.-H. The effect of cholesterol in the liposome bilayer on the stabilization of incorporated retinol. J. Liposome Res. 2005, 15, 157-166.

(30) Drujan, B. D.; Castillon, R.; Guerrero, E. Application of fluorometry in determination of vitamin A. Anal. Biochem. 1968, 23, $44-52$.

(31) Furr, H. C. Analysis of retinoids and carotenoids: problems resolved and unsolved. J. Nutr. 2004, 134, 281S-285S.

(32) Traikia, M.; Warschawski, D. E.; Recouvreur, M.; Cartaud, J.; Devaux, P. F. Formation of unilamellar vesicles by repetitive freezethaw cycles: characterization by electron microscopy and 31P-nuclear magnetic resonance. Eur. Biophys. J. 2000, 29, 184-195.

(33) Fex, G.; Johannesson, G. Studies of the spontaneous transfer of retinol from the retinol: retinol-binding protein complex to unilamellar liposomes. Biochim. Biophys. Acta, Biomembr. 1987, 901, 255-264.

(34) Fex, G.; Johannesson, G. Retinol transfer across and between phospholipid bilayer membranes. Biochim. Biophys. Acta, Biomembr. 1988, 944, 249-255.

(35) Fex, G.; Johannesson, G. [42] Transfer of retinol from retinolbinding protein complex to liposomes and across liposomal membranes. Methods Enzymol. 1990, 189, 394-402.

(36) Kian, R.; Seyed Ahmadian, S. M.; Zakerhamidi, M. S. Photophysical properties of different types of vitamin A in solvent media. J. Mol. Struct. 2015, 1080, 8-13.

(37) Georghiou, S.; Churchich, J. E. Nanosecond spectroscopy of retinol. J. Biol. Chem. 1975, 250, 1149-1151. 
(38) Werker, A.; Jaggard, K. W. Modelling Asymmetrical Growth Curves that Rise and then Fall: Applications to Foliage Dynamics of Sugar Beet (Beta vulgaris L.). Ann. Bot. 1997, 79, 657-665.

(39) Stratonnikov, A. A.; Meerovich, G. A.; Loschenov, V. B. Photobleaching of Photosensitizers Applied for Photodynamic Therapy. Proceedings of SPIE-The International Society for Optical Engineering, 2000, p 3909.

(40) Georgakoudi, I.; Nichols, M. G.; Foster, T. H. The mechanism of Photofrin photobleaching and its consequences for photodynamic dosimetry. Photochem. Photobiol. 1997, 65, 135-144.

(41) Samkoe, K. S.; Fecica, M. S.; Goyan, R. L.; Buchholz, J. L.; Campbell, C.; Kelly, N. M.; Cramb, D. T. Photobleaching kinetics of optically trapped multilamellar vesicles containing verteporfin using two-photon excitation section sign. Photochem. Photobiol. 2006, 82, $152-157$.

(42) Huff, J. The Fast mode for ZEISS LSM 880 with Airyscan: high-speed confocal imaging with super-resolution and improved signal-to-noise ratio. Nat. Methods 2016, 13, I-II.

(43) Imanishi, Y.; Palczewski, K. Visualization of Retinoid Storage and Trafficking by Two-Photon Microscopy. Retinoids: Methods and Protocols, 2010; Vol. 652, pp 247-261.

(44) Palczewska, G.; Golczak, M.; Williams, D. R.; Hunter, J. J.; Palczewski, K. Endogenous Fluorophores Enable Two-Photon Imaging of the Primate Eye. Invest. Ophthalmol. Visual Sci. 2014, 55, 4438-4447.

(45) Schindelin, J.; Arganda-Carreras, I.; Frise, E.; Kaynig, V.; Longair, M.; Pietzsch, T.; Preibisch, S.; Rueden, C.; Saalfeld, S.; Schmid, B.; Tinevez, J.-Y.; White, D. J.; Hartenstein, V.; Eliceiri, K.; Tomancak, P.; Cardona, A. Fiji: an open-source platform for biological-image analysis. Nat. Methods 2012, 9, 676-682. 